\title{
A Molecular Switch with pH-Controlled Absolutely Switchable Dual-Mode fluorescence
}

Yasuhiro Shiraishi,* Yasufumi Tokitoh, Go Nishimura and Takayuki Hirai

Research Center for Solar Energy Chemistry, and Division of Chemical Engineering, Graduate School of Engineering Science, Osaka University, Toyonaka 560-8531, Japan

E-mail: shiraish@cheng.es.osaka-u.ac.jp

\section{Supporting Information}

Table S1. Stepwise protonation constants of $\mathbf{L} 1-\mathbf{L} 4$, determined in aqueous $\mathrm{NaCl}(0.15 \mathrm{M})$ solution at $298 \mathrm{~K}^{\mathrm{a}}$

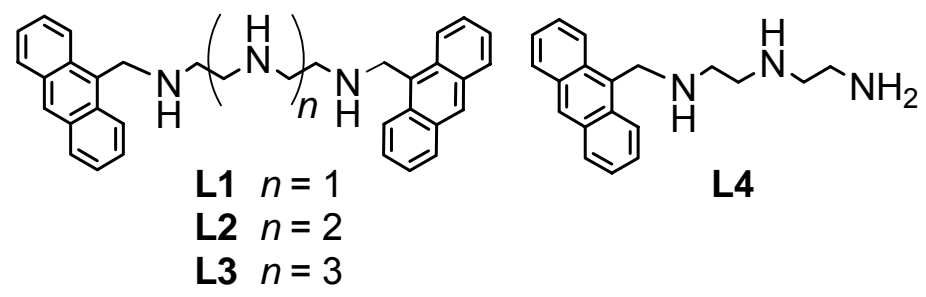

\begin{tabular}{ccccc}
\hline Reaction $^{b}$ & L1 & L2 & L3 & L4 \\
\hline $\mathrm{H}+\mathrm{L}=\mathrm{HL}$ & $9.45(1)$ & $9.72(1)$ & $9.02(1)$ & $9.42(1)$ \\
$\mathrm{H}+\mathrm{HL}=\mathrm{H}_{2} \mathrm{~L}$ & $7.55(2)$ & $8.52(1)$ & $8.45(1)$ & $7.42(1)$ \\
$\mathrm{H}+\mathrm{H}_{2} \mathrm{~L}=\mathrm{H}_{3} \mathrm{~L}$ & $6.01(3)$ & $7.08(2)$ & $6.78(2)$ & $4.39(2)$ \\
$\mathrm{H}+\mathrm{H}_{3} \mathrm{~L}=\mathrm{H}_{4} \mathrm{~L}$ & & $5.31(4)$ & $4.54(3)$ & \\
$\mathrm{H}+\mathrm{H}_{4} \mathrm{~L}=\mathrm{H}_{5} \mathrm{~L}$ & & & $3.45(4)$ & \\
$\log \beta$ & 23.01 & 30.63 & 32.24 & 21.23 \\
\hline
\end{tabular}

${ }^{a}$ Numbers in parentheses are standard deviations in the last significant figure.

${ }^{b}$ Charges are omitted for clarity. 


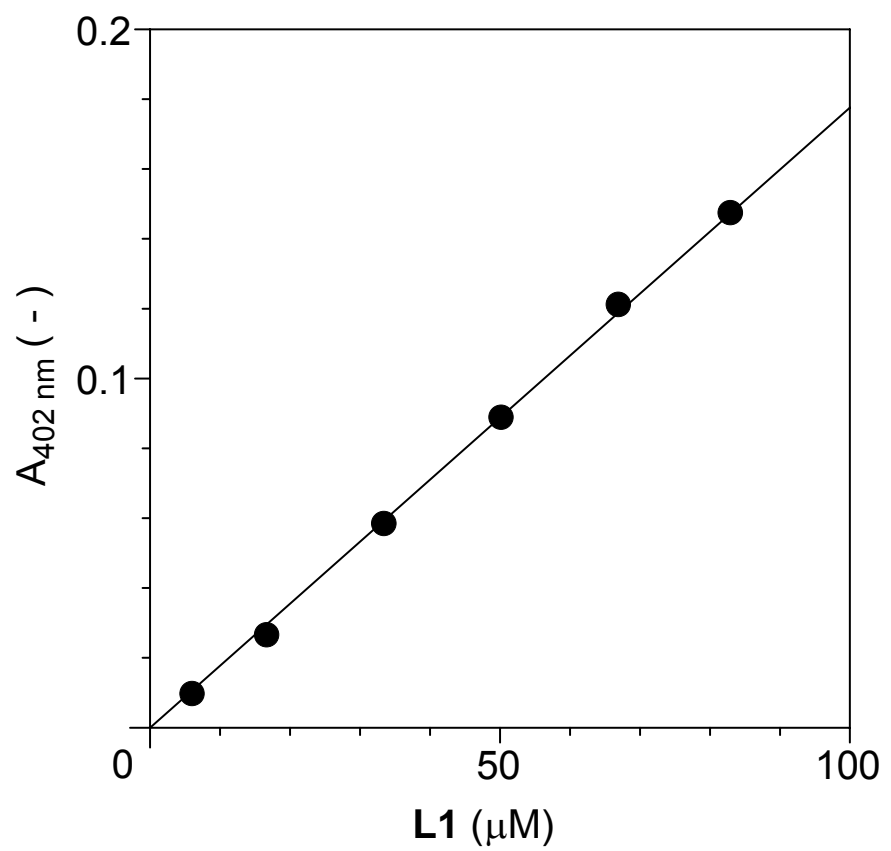

Figure S1. The Beer's Law plot between the L1 concentration in water (pH 13.0 at $298 \mathrm{~K}$ ) and absorbance at $402 \mathrm{~nm}$. 

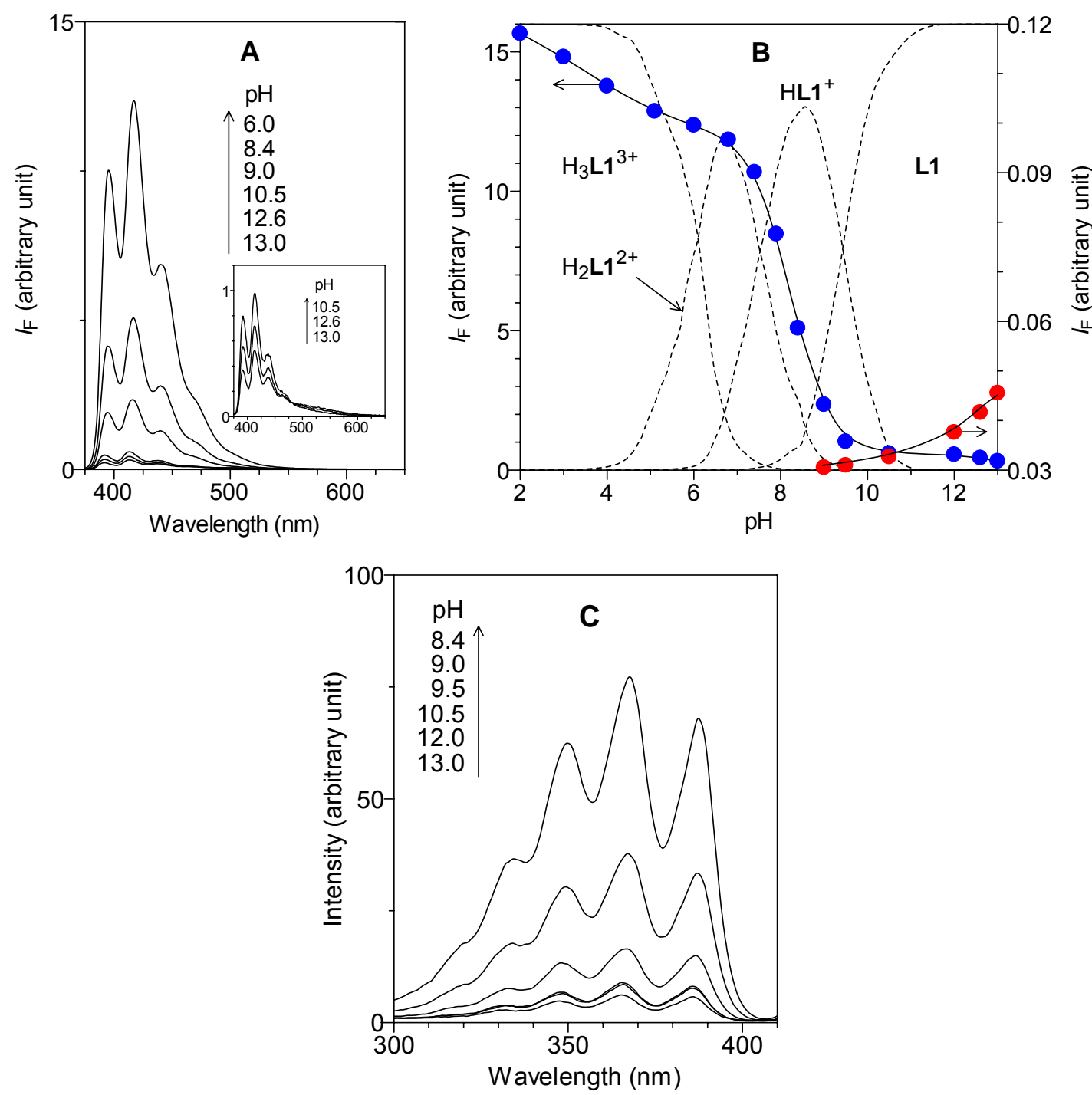

Figure S2. (A) Fluorescence spectra $\left(\lambda_{\mathrm{ex}}=368 \mathrm{~nm}\right)$ of $\mathbf{L} 1(70 \mu \mathrm{M})$ in aqueous $\mathrm{NaCl}(0.15 \mathrm{M})$ solution at $298 \mathrm{~K}$. (B) Mole fraction distribution of the protonation states of L1 (dotted line) and intensity of the fluorescence emissions $\left(\lambda_{\mathrm{ex}}=368 \mathrm{~nm}\right)$ at $\lambda_{\mathrm{em}}=416 \mathrm{~nm}$ (blue) and $520 \mathrm{~nm}$ (red). (C) Excitation spectra of $\mathbf{L} 1\left(\lambda_{\mathrm{em}}=416 \mathrm{~nm}\right)$ in aqueous $\mathrm{NaCl}(0.15 \mathrm{M})$ solution at $298 \mathrm{~K}$. 

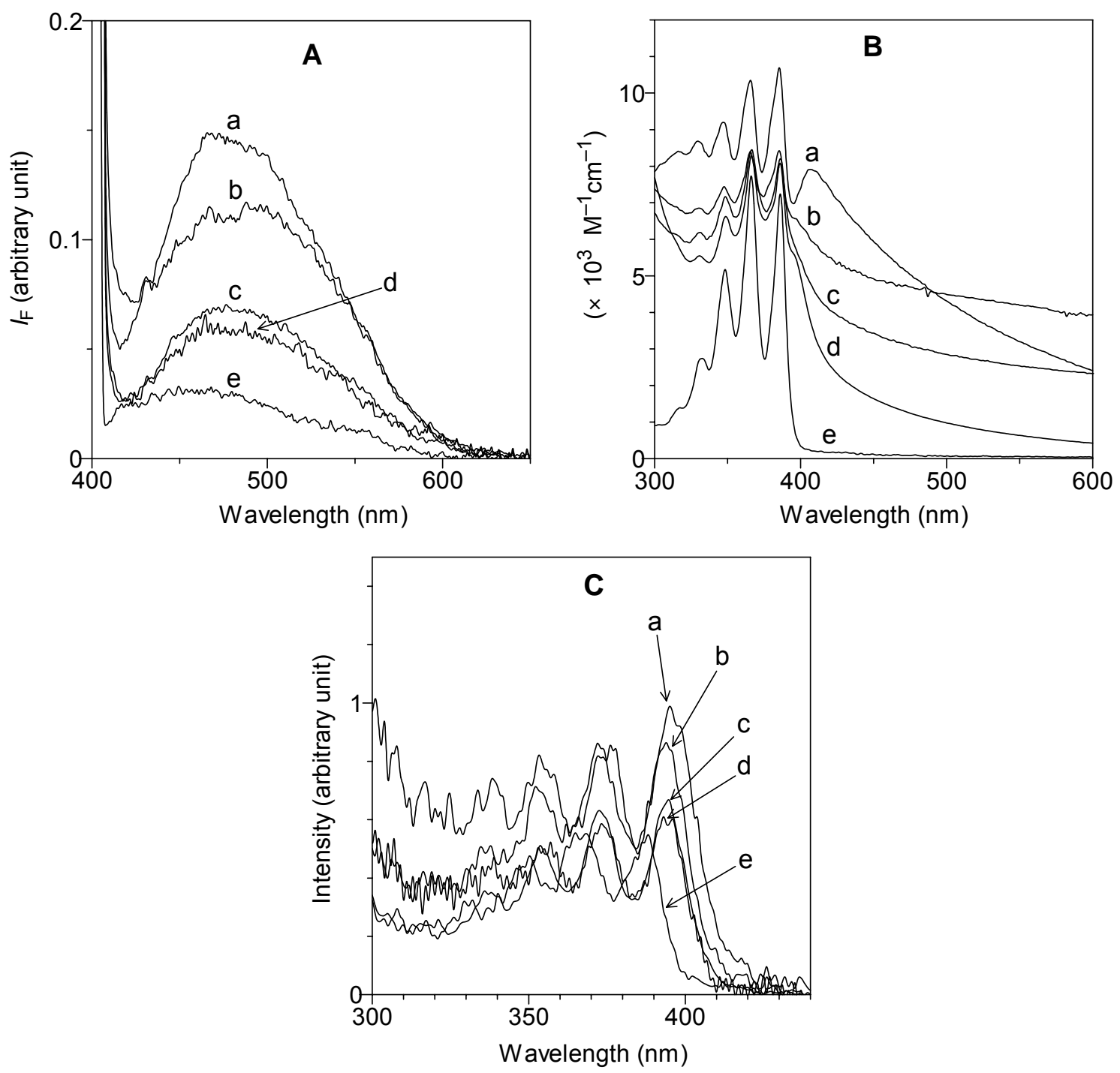

Figure S3. (A) Fluorescence $\left(\lambda_{\mathrm{ex}}=402 \mathrm{~nm}\right),(\mathbf{B})$ absorption, and $(\mathbf{C})$ excitation spectra $\left(\lambda_{\mathrm{em}}=520\right.$ $\mathrm{nm})$ of $\mathbf{L 1}(70 \mu \mathrm{M})$ dissolved in various solvents of $\mathrm{pH} 13.0$ at $298 \mathrm{~K}$. The respective solvents are: (a) water, (b) water/acetonitrile $(9 / 1 \mathrm{v} / \mathrm{v}),(\mathbf{c}) \mathrm{D}_{2} \mathrm{O}$, (d) water/acetonitrile $(7 / 1 \mathrm{v} / \mathrm{v})$, and (e) water/acetonitrile $(1 / 1 \mathrm{v} / \mathrm{v})$. 

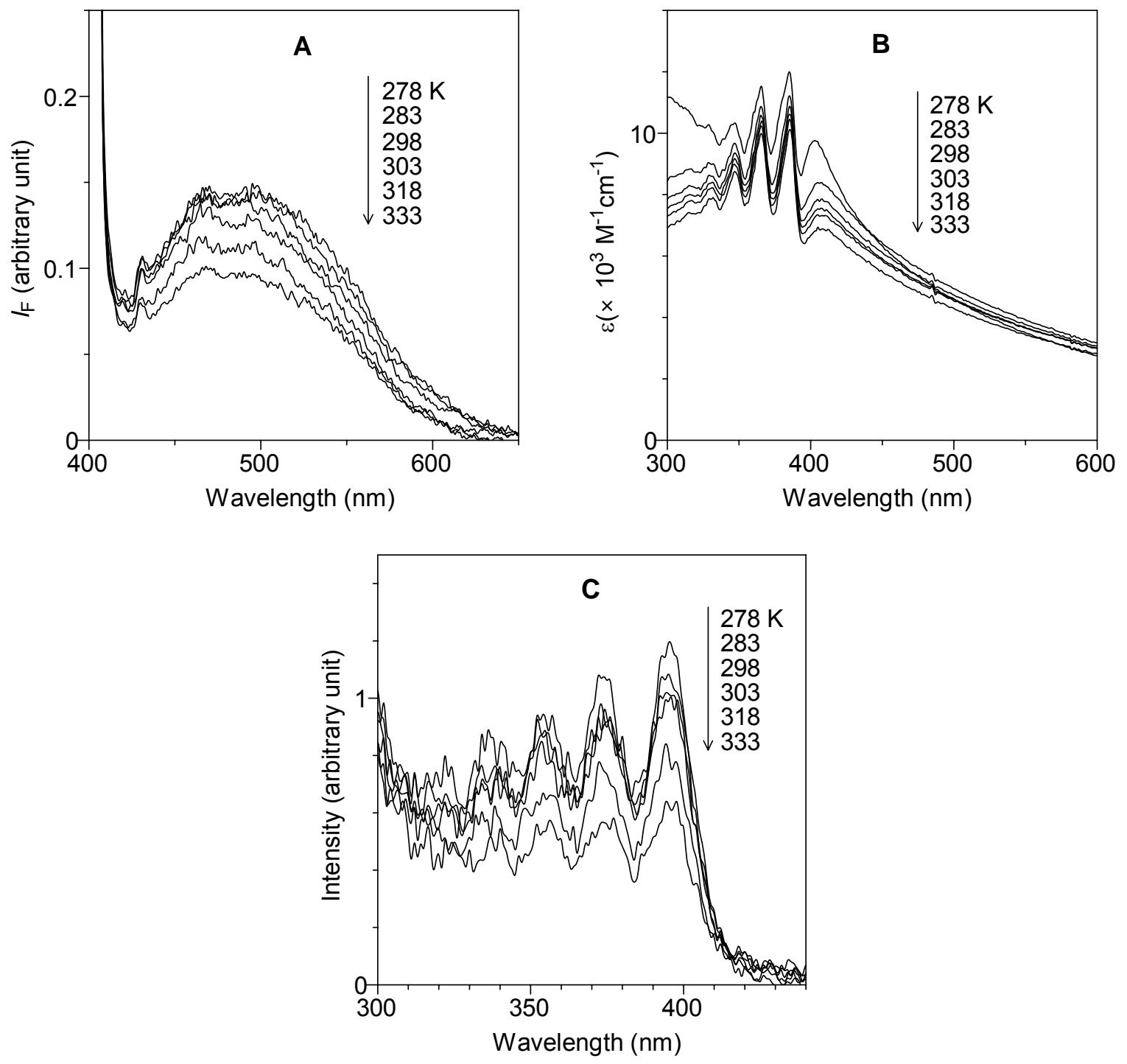

Figure S4. Changes in (A) fluorescence $\left(\lambda_{\mathrm{ex}}=402 \mathrm{~nm}\right),(\mathbf{B})$ absorption, and (C) excitation spectra $\left(\lambda_{\mathrm{em}}=520 \mathrm{~nm}\right)$ of $\mathbf{L} \mathbf{1}(70 \mu \mathrm{M})$ in water $(\mathrm{pH} 13.0)$ with temperature. 

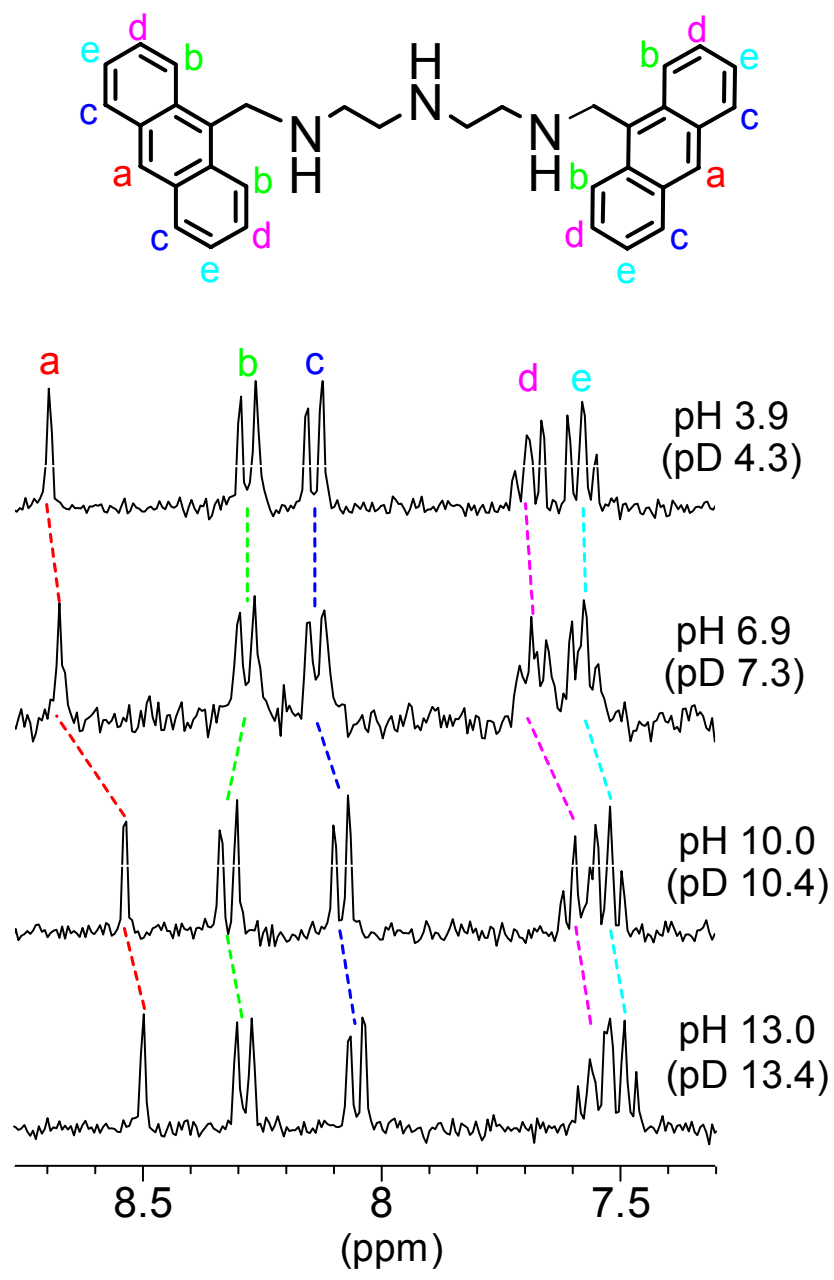

Figure S5. ${ }^{1} \mathrm{H}$ NMR spectra of $\mathrm{L} 1$ in $\mathrm{D}_{2} \mathrm{O} \cdot \mathrm{pH}(=\mathrm{pD}-0.4)$ of the respective solution was adjusted with $\mathrm{DCl}$ and $\mathrm{NaOD}$. 

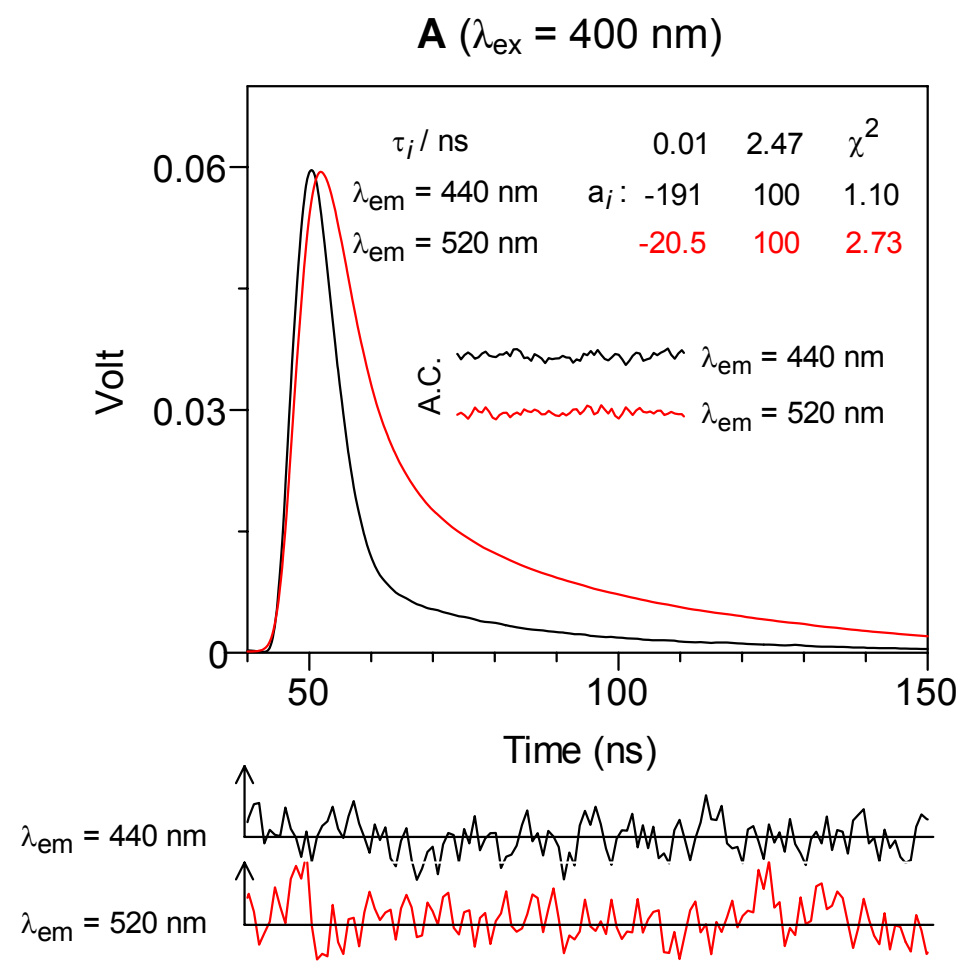

Weighted residuals

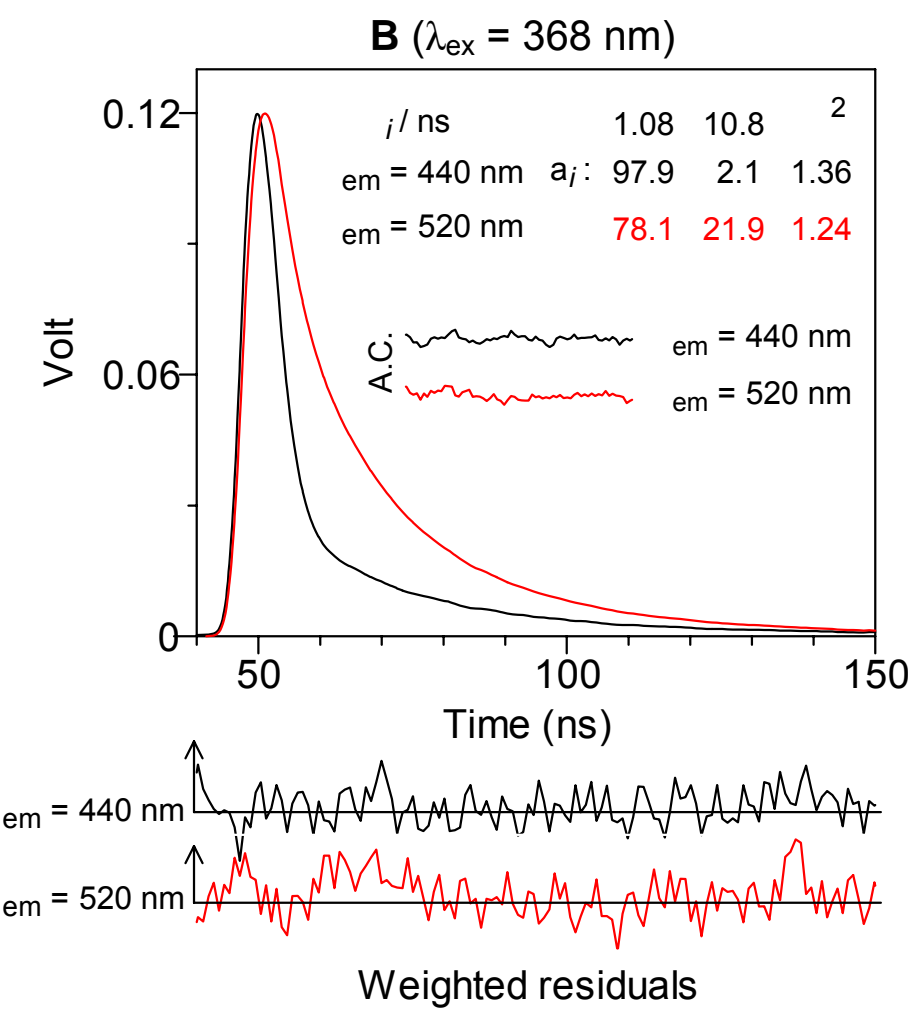

Figure S6. Fluorescence decays and pulse instrumental response of L1 ( $\mathrm{pH} 13.0)$, when excited at (A) $400 \mathrm{~nm}$ and (B) $368 \mathrm{~nm}$. Shown as insert are the decay times $\left(\tau_{i} / \mathrm{ns}\right)$ and the normalized pre-exponential factors $\left(a_{i}\right)$. For judging the quality of the fit, chi-squares $\left(\chi^{2}\right)$, autocorrelation functions (A.C.), and weighted residuals are also shown in the figure. 
Table S2. Fluorescence decay times $\left(\tau_{i}\right)$ and pre-exponential factors $\left(a_{i}\right)$ for L1 as a function of $\mathrm{pH}$, when excited at $\lambda_{\mathrm{ex}}=400 \mathrm{~nm}^{2}$

\begin{tabular}{|c|c|c|c|c|c|c|c|}
\hline $\mathrm{pH}$ & $\begin{array}{c}\lambda_{\mathrm{em}} \\
(\mathrm{nm})\end{array}$ & $\begin{array}{c}\tau_{1} / \mathrm{ns} \\
\left(\mathrm{a}_{1}\right) \\
\end{array}$ & $\begin{array}{c}\tau_{2} / \mathrm{ns} \\
\left(\mathrm{a}_{2}\right) \\
\end{array}$ & $\begin{array}{c}\tau_{3} / \mathrm{ns} \\
\left(\mathrm{a}_{3}\right) \\
\end{array}$ & $\begin{array}{c}\tau_{4} / \mathrm{ns} \\
\left(\mathrm{a}_{4}\right) \\
\end{array}$ & $\begin{array}{c}\tau_{5} / \mathrm{ns} \\
\left(\mathrm{a}_{5}\right) \\
\end{array}$ & $\chi^{2}$ \\
\hline \multirow{2}{*}{1.72} & 440 & $\begin{array}{l}1.415 \\
(100) \\
\end{array}$ & & & & & 3.44 \\
\hline & 520 & $\begin{array}{l}1.415 \\
(100)\end{array}$ & & & & & 1.599 \\
\hline \multirow{2}{*}{5.93} & 440 & $\begin{array}{c}1.415 \\
(9.508) \\
\end{array}$ & $\begin{array}{c}2.48 \\
(88.92) \\
\end{array}$ & $\begin{array}{c}5.653 \\
(1.569) \\
\end{array}$ & & & 2.496 \\
\hline & 520 & $\begin{array}{c}1.415 \\
(31.73)\end{array}$ & $\begin{array}{c}2.48 \\
(59.03)\end{array}$ & $\begin{array}{c}5.653 \\
(9.239)\end{array}$ & & & 2.200 \\
\hline \multirow{2}{*}{6.76} & 440 & & $\begin{array}{c}2.48 \\
(79.48)\end{array}$ & $\begin{array}{c}5.653 \\
(20.52)\end{array}$ & & & 8.296 \\
\hline & 520 & & $\begin{array}{c}2.48 \\
(94.58)\end{array}$ & $\begin{array}{c}5.653 \\
(5.423)\end{array}$ & & & 1.459 \\
\hline \multirow{2}{*}{13.0} & 440 & & & & $\begin{array}{c}0.01 \\
(-191.4)\end{array}$ & $\begin{array}{l}2.469 \\
(100)\end{array}$ & 1.104 \\
\hline & 520 & & & & $\begin{array}{c}0.01 \\
(-20.54)\end{array}$ & $\begin{array}{l}2.469 \\
(100)\end{array}$ & 2.731 \\
\hline
\end{tabular}

a $\tau_{1}, \mathrm{a}_{1}$ : for excited monomer $\mathrm{H}_{3} \mathbf{L} \mathbf{1}^{3+}$ species. $\tau_{2}, \mathrm{a}_{2}$ : for excited monomer $\mathrm{H}_{2} \mathbf{L} \mathbf{1}^{2+}$ species. $\tau_{3}, a_{3}$ : for excited monomer $\mathrm{HL1}^{+}$species. $\tau_{4}, \mathrm{a}_{4}$ : for excited monomer $\mathbf{L} \mathbf{1}$ species $\left({ }^{1} \mathbf{M}^{*}\right)$. $\tau_{5}, a_{5}$ for excimer species $\left({ }^{1} \mathrm{E}^{*}\right)$.

Table S3. Fluorescence decay times $\left(\tau_{i}\right)$ and pre-exponential factors $\left(a_{i}\right)$ for L1 as a function of $\mathrm{pH}$, when excited at $\lambda_{\mathrm{ex}}=368 \mathrm{~nm}^{a}$

\begin{tabular}{|c|c|c|c|c|c|c|c|}
\hline $\mathrm{pH}$ & $\begin{array}{c}\lambda_{\mathrm{em}} \\
(\mathrm{nm})\end{array}$ & $\begin{array}{c}\tau_{1} / \mathrm{ns} \\
\left(\mathrm{a}_{1}\right)\end{array}$ & $\begin{array}{c}\tau_{2} / \mathrm{ns} \\
\left(\mathrm{a}_{2}\right)\end{array}$ & $\begin{array}{c}\tau_{3} / \mathrm{ns} \\
\left(\mathrm{a}_{3}\right)\end{array}$ & $\begin{array}{l}\tau_{4} / \mathrm{ns} \\
\left(\mathrm{a}_{4}\right)\end{array}$ & $\begin{array}{c}\tau_{5} / \mathrm{ns} \\
\left(\mathrm{a}_{5}\right)\end{array}$ & $\chi^{2}$ \\
\hline \multirow{2}{*}{1.72} & 440 & $\begin{array}{l}10.75 \\
(100)\end{array}$ & & & & & 3.797 \\
\hline & 520 & $\begin{array}{l}10.75 \\
(100)\end{array}$ & & & & & 1.515 \\
\hline \multirow{2}{*}{5.93} & 440 & $\begin{array}{c}10.75 \\
(22.06) \\
\end{array}$ & $\begin{array}{c}10.35 \\
(77.94) \\
\end{array}$ & & & & 16.82 \\
\hline & 520 & $\begin{array}{c}10.75 \\
(1.524) \\
\end{array}$ & $\begin{array}{c}10.35 \\
(98.48) \\
\end{array}$ & & & & 1.664 \\
\hline \multirow{2}{*}{8.52} & 440 & & & $\begin{array}{c}8.967 \\
(44.25) \\
\end{array}$ & $\begin{array}{c}1.082 \\
(55.08) \\
\end{array}$ & $\begin{array}{c}10.76 \\
(0.672)\end{array}$ & 2.261 \\
\hline & 520 & & & $\begin{array}{c}8.967 \\
(21.03) \\
\end{array}$ & $\begin{array}{c}1.082 \\
(63.62) \\
\end{array}$ & $\begin{array}{c}10.76 \\
(15.34) \\
\end{array}$ & 2.987 \\
\hline \multirow{2}{*}{13.0} & 440 & & & & $\begin{array}{c}1.082 \\
(97.87) \\
\end{array}$ & $\begin{array}{c}10.76 \\
(2.125) \\
\end{array}$ & 1.356 \\
\hline & 520 & & & & $\begin{array}{c}1.082 \\
(78.08)\end{array}$ & $\begin{array}{c}10.76 \\
(21.92)\end{array}$ & 1.235 \\
\hline
\end{tabular}

${ }^{a} \tau_{1}, \mathrm{a}_{1}$ : for excited monomer $\mathrm{H}_{3} \mathbf{L} \mathbf{1}^{3+}$ species. $\tau_{2}, \mathrm{a}_{2}$ : for excited monomer $\mathrm{H}_{2} \mathbf{L} \mathbf{1}^{2+}$ species. $\tau_{3}, \mathrm{a}_{3}$ : for excited monomer $\mathrm{HLI}^{+}$species. $\tau_{4}, \mathrm{a}_{4}$ : for excited monomer $\mathbf{L} \mathbf{1}$ species $\left({ }^{1} \mathrm{M}^{*}\right)$. $\tau_{5}, a_{5}$ : for excimer species $\left({ }^{1} \mathrm{E}^{*}\right)$. 

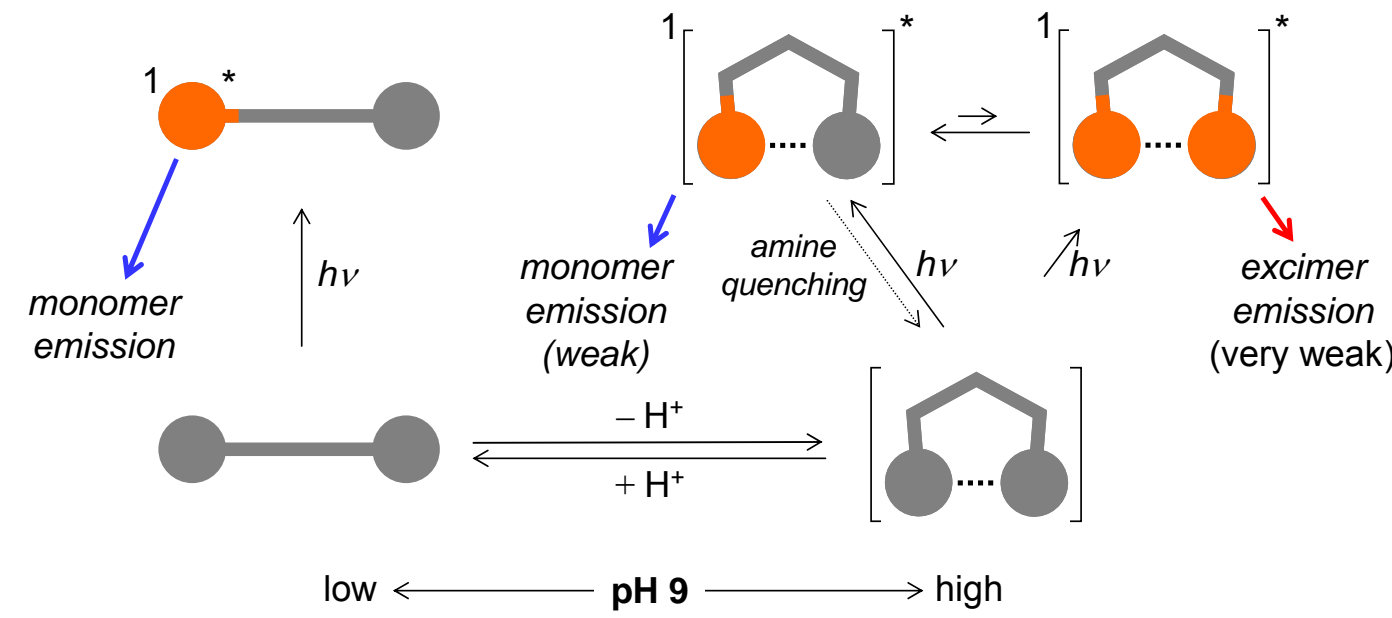

Figure S7. Emission Mechanism of L1 when excited at $\lambda_{\mathrm{ex}}=368 \mathrm{~nm}$. 

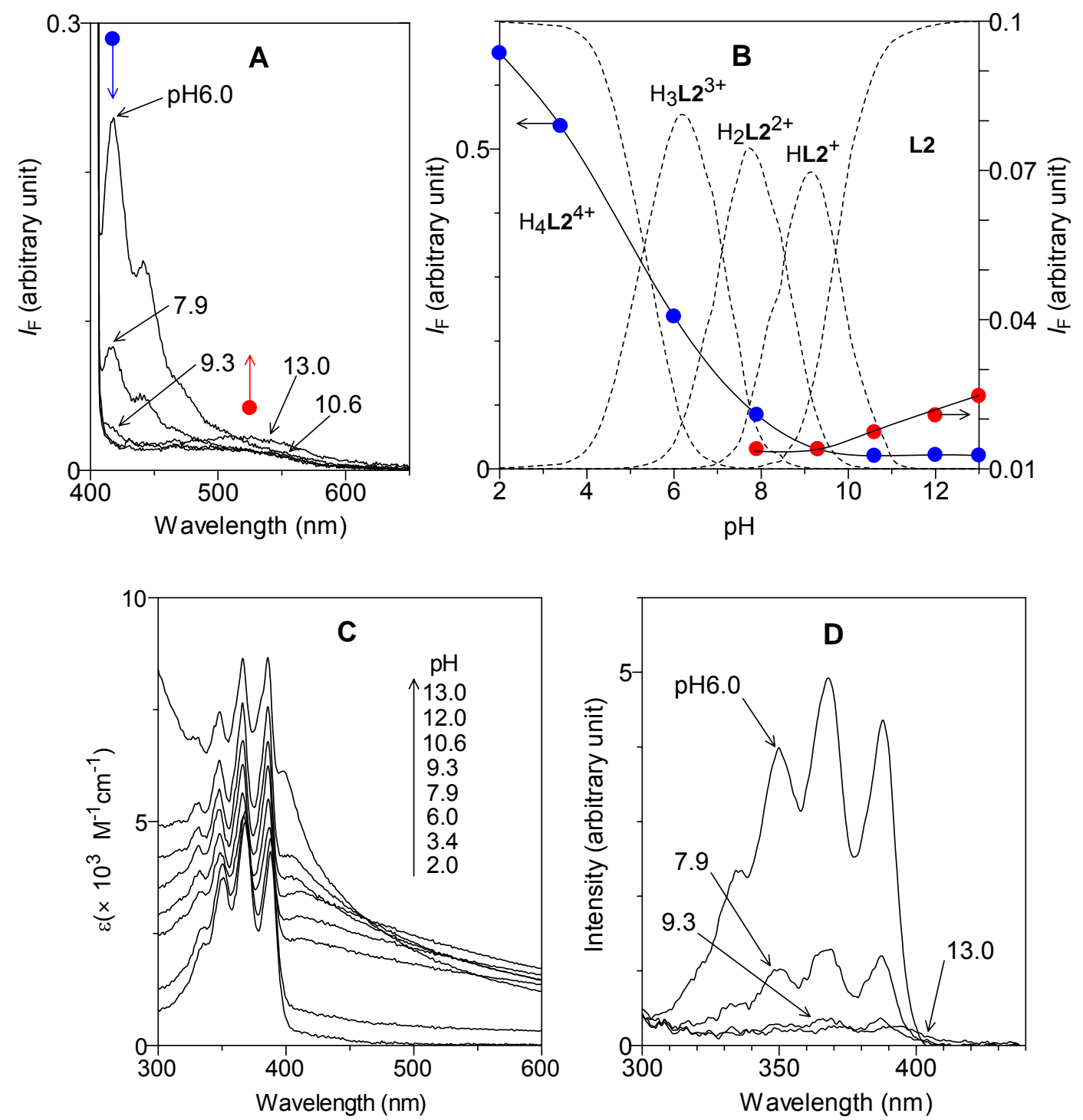

Figure S8. (A) Fluorescence spectra $\left(\lambda_{\text {ex }}=402 \mathrm{~nm}\right)$ of $\mathbf{L} 2(70 \mu \mathrm{M})$ in aqueous $\mathrm{NaCl}(0.15 \mathrm{M})$ solution at $298 \mathrm{~K}$. (B) Mole fraction distribution of the protonation states of L2 (dotted line) and intensity of the fluorescence emissions at $\lambda_{\mathrm{em}}=416 \mathrm{~nm}$ (blue) and $520 \mathrm{~nm}$ (red). (C) Absorption and $(\mathbf{D})$ excitation spectra $\left(\lambda_{\mathrm{em}}=520 \mathrm{~nm}\right)$ of $\mathbf{L} 2$ in aqueous $\mathrm{NaCl}(0.15 \mathrm{M})$ solution at $298 \mathrm{~K}$. 

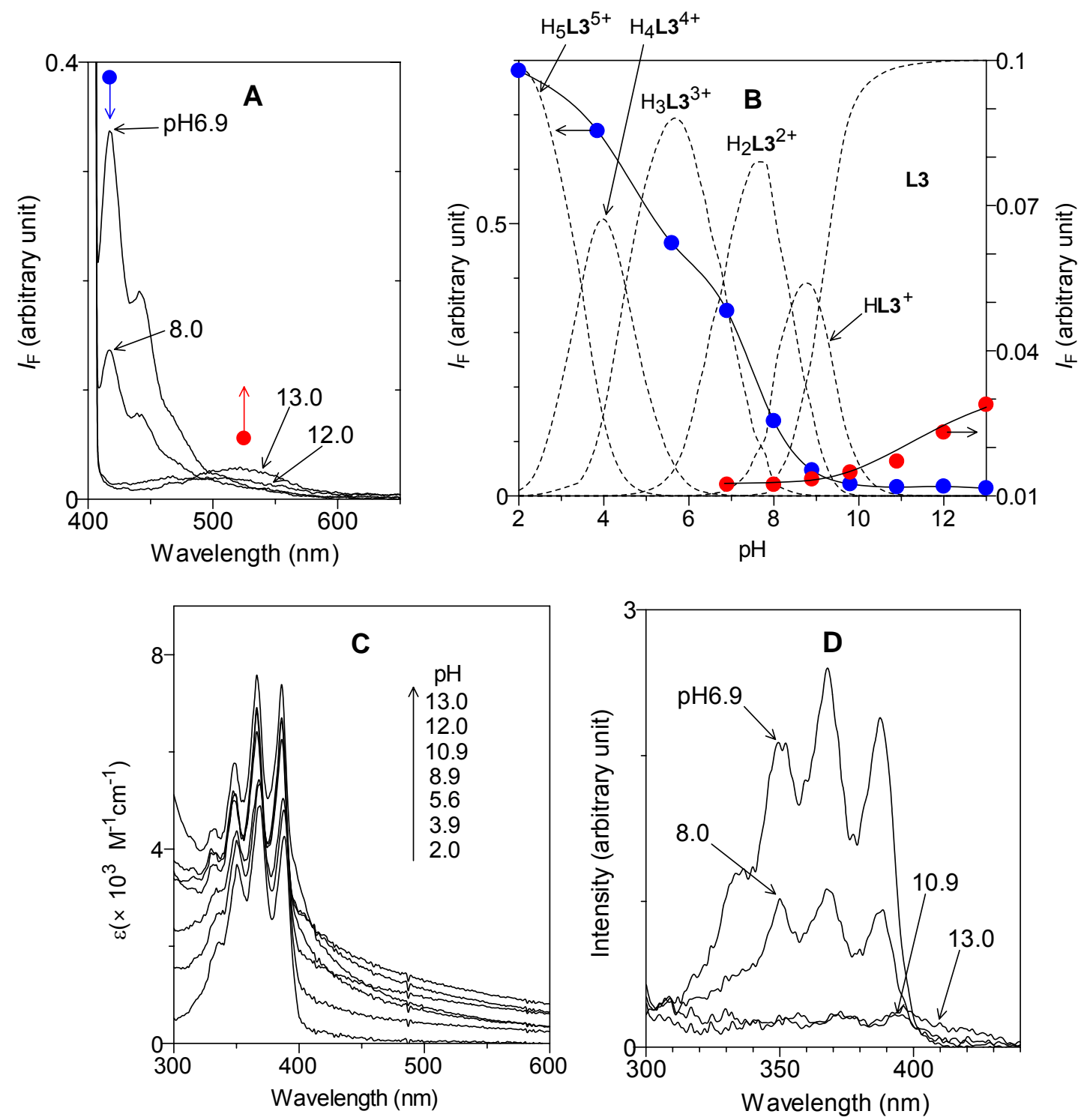

Figure S9. (A) Fluorescence spectra $\left(\lambda_{\mathrm{ex}}=402 \mathrm{~nm}\right)$ of $\mathbf{L 3}(70 \mu \mathrm{M})$ in aqueous $\mathrm{NaCl}(0.15 \mathrm{M})$ solution at $298 \mathrm{~K}$. (B) Mole fraction distribution of the protonation states of L3 (dotted line) and intensity of the fluorescence emissions at $\lambda_{\mathrm{em}}=416 \mathrm{~nm}$ (blue) and $520 \mathrm{~nm}$ (red). (C) Absorption and (D) excitation spectra $\left(\lambda_{\mathrm{em}}=520 \mathrm{~nm}\right)$ of $\mathbf{L 3}$ in aqueous $\mathrm{NaCl}(0.15 \mathrm{M})$ solution at $298 \mathrm{~K}$. 

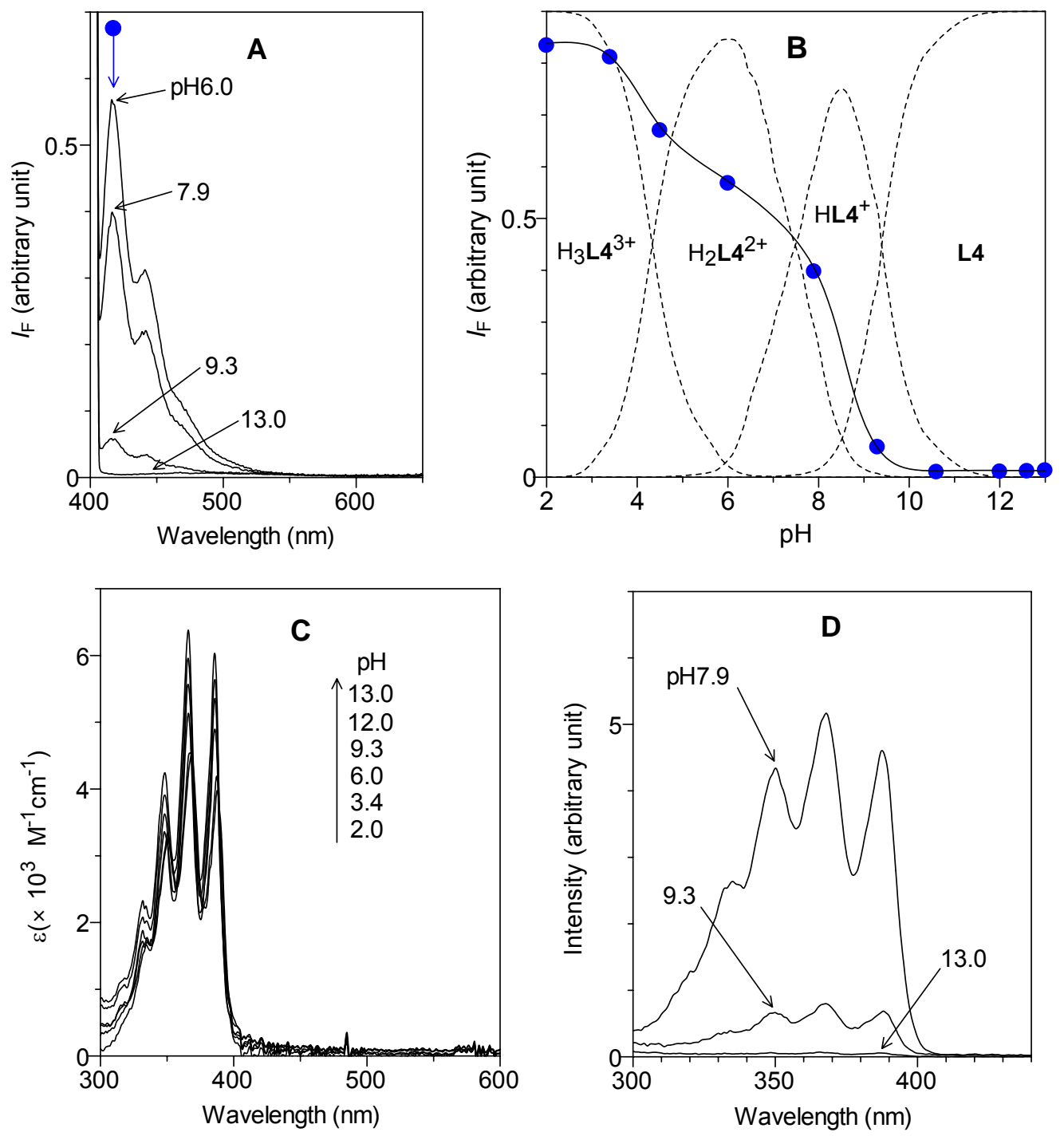

Figure S10. (A) Fluorescence spectra $\left(\lambda_{\mathrm{ex}}=402 \mathrm{~nm}\right)$ of $\mathbf{L} 4(70 \mu \mathrm{M})$ in aqueous $\mathrm{NaCl}(0.15 \mathrm{M})$ solution at $298 \mathrm{~K}$. (B) Mole fraction distribution of the protonation states of L4 (dotted line) and intensity of the fluorescence emission at $\lambda_{\mathrm{em}}=416 \mathrm{~nm}$ (blue). (C) Absorption and (D) excitation spectra $\left(\lambda_{\text {em }}=520 \mathrm{~nm}\right)$ of $\mathbf{L} 4$ in aqueous $\mathrm{NaCl}(0.15 \mathrm{M})$ solution at $298 \mathrm{~K}$. 